\title{
Regulation of Drug Metabolism by the Interplay of Inflammatory Signaling, Steatosis, and Xeno-Sensing Receptors in HepaRG Cells ${ }^{\mathbb{}}$
}

\author{
Norman Tanner, Lisa Kubik, Claudia Luckert, Maria Thomas, Ute Hofmann, Ulrich M. Zanger, \\ Linda Böhmert, Alfonso Lampen, and Albert Braeuning
}

German Federal Institute for Risk Assessment, Department Food Safety, Berlin (N.T., L.K., C.L., L.B., A.L., A.B.), and Dr. Margarete
Fischer-Bosch-Institute for Clinical Pharmacology, Stuttgart (M.T., U.H., U.M.Z.), Germany

Received September 27, 2017; accepted January 11, 2018

\section{ABSTRACT}

Nonalcoholic fatty liver disease (NAFLD), which is characterized by triglyceride deposition in hepatocytes resulting from imbalanced lipid homeostasis, is of increasing concern in Western countries, along with progression to nonalcoholic steatohepatitis (NASH), liver fibrosis, and cirrhosis. Previous studies suggest a complex, mutual influence of hepatic fat accumulation, NASH-related inflammatory mediators, and drug-sensing receptors regulating xenobiotic metabolism. Here, we investigated the suitability of human HepaRG hepatocarcinoma cells as a model for NAFLD and NASH. Cells were incubated for up to 14 days with an oleate/palmitate mixture (125 $\mu \mathrm{M}$ each) and/or with $10 \mathrm{ng} / \mathrm{ml}$ of the inflammatory mediator interleukin6 (IL-6). Effects of these conditions on the regulation of drug metabolism were studied using xenobiotic agonists of the aryl hydrocarbon receptor (AHR), pregnane $X$ receptor (PXR), constitutive androstane receptor (CAR), nuclear factor (erythroid-derived 2)- like 2, and peroxisome proliferator-activated receptor $\alpha$ (PPAR $\alpha$ ). Results underpin the suitability of HepaRG cells for NAFLD- and NASH-related research and constitute a broad-based analysis of the impact of hepatic fatty acid accumulation and inflammation on drug metabolism and its inducibility by xenobiotics. IL-6 exerted pronounced negative regulatory effects on basal as well as on PXR-, CAR-, and PPAR $\alpha$-, but not AHR-dependent induction of drugmetabolizing enzymes. This inhibition was related to diminished transactivation potential of the respective receptors rather than to reduced transcription of nuclear receptor-encoding mRNAs. The most striking effects of IL-6 and/or fatty acid treatment were observed in HepaRG cells after 14 days of treatment, making these cultures appear a suitable model for studying the relationship of fatty acid accumulation, inflammation, and xenobiotic-induced drug metabolism.

\section{Introduction}

A major part of drug and xenobiotic metabolism is carried out in the liver. The expression and activity of many drug-metabolizing enzymes are regulated via ligand-activated nuclear receptors, which function as sensors for xenobiotics. In particular, induction of cytochrome P450 (P450) enzymes by exogenous compounds via the aryl hydrocarbon receptor (AHR), constitutive androstane receptor (CAR), and pregnane $\mathrm{X}$ receptor (PXR) has been studied extensively (Honkakoski and Negishi, 2000). In addition to xenobiotic nuclear receptor ligands, endogenous signaling pathways also affect the regulation of hepatic drug metabolism via nuclear receptors [e.g., see Braeuning et al. (2009), Braeuning and Schwarz (2010), or Schulthess et al. (2015)].

This work was supported by the German Federal Institute for Risk Assessment (BfR) [Grant no. 1322-625] and by the Robert Bosch Foundation, Stuttgart, Germany.

https://doi.org/10.1124/dmd.117.078675.

S This article has supplemental material available at dmd.aspetjournals.org.
Nonalcoholic fatty liver disease (NAFLD) as the hepatic manifestation of the metabolic syndrome is characterized by the (generally reversible) deposition of triglycerides in hepatocytes resulting from multiple imbalances in lipid homeostasis (Day and James, 1998). NAFLD is of increasing concern in Western countries, and it is believed that, as a result of unbalanced dietary intake of fats and carbohydrates, up to one quarter of the population is affected (Weiß et al., 2014). NAFLD may progress to nonalcoholic steatohepatitis (NASH), which is linked to an increase in proinflammatory cytokines in the liver, thereby leading to inflammation and oxidative stress and apoptosis of individual hepatocytes (Day and James, 1998; Hijona et al., 2010). The exact molecular determinants that trigger the transition from NAFLD to NASH are still not fully understood. Perpetuated hepatic cell death and regenerative proliferation in inflamed livers might subsequently give rise to the onset of irreversible hepatic fibrosis and cirrhosis. The latter is considered a major risk factor for the development of hepatocellular carcinoma (Sanyal et al., 2010; Toosi, 2015).

Published data indicate interactions between NAFLD and drug metabolism. Different nuclear receptors, as well as drug-metabolizing

ABBREVIATIONS: AHR, aryl hydrocarbon receptor; ANOVA, analysis of variance; CAR, constitutive androstane receptor; CITCO, 6-(4chlorophenyl)imidazo[2,1-b][1,3]thiazole-5-carbaldehyde O-(3,4-dichlorobenzyl)oxime; DMSO, dimethyl sulfoxide; HNF, hepatocyte nuclear factor; IL, interleukin; LXR, liver X receptor; 3-MC, 3-methylcholanthrene; NAFLD, nonalcoholic fatty liver disease; NASH, nonalcoholic steatohepatitis; O/P, equimolar oleate/palmitate mixture; P450, cytochrome P450; PPAR, peroxisome proliferator-activated receptor; PXR, pregnane X receptor; RT-PCR, reverse transcription-polymerase chain reaction; tBHQ, tert-butylhydroquinone; Wy14,643, 4-chloro-6-(2,3-xylidino)-2-pyrimidinylthioacetic acid. 
enzymes, have been identified as being involved in the formation of liver steatosis based on animal models or patient data [for details, see the review by Naik et al. (2013)]. P450 oxidoreductase knockout-driven loss of $\mathrm{P} 450$ activities in a transgenic mouse model affects hepatic lipid accumulation, as well as CAR and PXR activities (Finn et al., 2009). Alterations in the activity and abundance of various P450s and phase 2 enzymes in rodents and humans have been summarized by GómezLechón et al. (2009) and by Merrell and Cherrington (2011). Their conclusions indicate pleiotropic effects of NAFLD and NASH on drugmetabolizing enzymes; however, these effects are not always consistent with results on the upregulation or downregulation of certain P450 isoforms under different conditions of experimental animal research or clinical studies. Previous studies suggest a negative influence of steatosis on various $\mathrm{P} 450$ activities in human primary hepatocytes in vitro (Donato et al., 2006), whereas in HepaRG cells, prolonged PPAR $\alpha$ activation counteracts steatosis in these cells (Rogue et al., 2014). Inflammatory mediators, for example, interleukin 6 (IL-6), are known to broadly decrease the drug-metabolizing capacities of hepatocytes (Morgan et al., 2008). Mechanistically, this phenomenon results mainly from attenuated transcription of drug metabolism-related genes and seems to involve interference of inflammation signaling with the activity of hepatocyteenriched transcription factors from the hepatocyte nuclear factor (HNF) family, together with downregulation or inactivation of nuclear receptors engaged in the regulation of drug metabolism (Pascussi et al., 2000; Merrell and Cherrington, 2011; Liu et al., 2012; Klein et al., 2015).

Whereas primary hepatocytes are still considered a gold standard for in vitro research in liver metabolism (Godoy et al., 2013), their use is often limited by scarce availability and large interdonor variation. Human HepaRG hepatocarcinoma cells constitute an alternative to primary human hepatocytes because this permanent cell line can differentiate into hepatocyte-like cells, which possess many physiologic functions of hepatocytes, especially with regard to drug metabolism (Aninat et al., 2006). Therefore, HepaRG cells have been used successfully for in vitro experimentation in basic research and drug development for a few years. Some previous studies have used HepaRG cells as an in vitro model for hepatic steatosis (Brown et al., 2013; Rogue et al., 2014) or inflammation (Dubois-Pot-Schneider et al., 2014; Klein et al., 2015; Rubin et al., 2015), thus demonstrating the general suitability of this cell line for NAFLD and NASH research.
Previous studies have addressed selected endpoints, whereas comparative systematic analyses of basal and xenobiotic-induced drug metabolism in HepaRG cells under different experimental conditions representing NAFLD and NASH are still not available. The present study was aimed to characterize systematically the impact of steatotic and inflammatory conditions on the basal and inducible expression of drug-metabolizing enzymes in HepaRG cells.

\section{Materials and Methods}

Cell Culture and Treatment. HepaRG human hepatocarcinoma cells (Biopredic, Rennes, France) were differentiated and cultivated, as recently described (Luckert et al., 2017), including 2 weeks' incubation with $1.7 \%$ dimethyl sulfoxide (DMSO) for the induction of differentiation into hepatocytelike cells. Fetal calf serum concentrations were reduced to $1 \% 24$ hours before treatment with the compounds listed to follow. Standard two-dimensional cultivation of HepG2 human hepatocarcinoma cells has been described previously (Luckert et al., 2017). Cells were treated according to the scheme depicted in Fig. 1 with the following chemicals: xenobiotic-sensing receptors were activated by treatment with either $5 \mu \mathrm{M}$ AHR agonist 3-methylcholanthrene (3-MC; Enzo Life Sciences, Lörrach, Germany), $5 \mu \mathrm{M}$ CAR agonist 6-(4-chlorophenyl)imidazo[2,1-b][1,3]thiazole-5-carbaldehyde-O-(3,4-dichlorobenzyl)oxime (CITCO; Enzo Life Sciences), $10 \mu \mathrm{M}$ PXR agonist rifampicin (Rif; Sigma, Taufkirchen, Germany), $30 \mu \mathrm{M}$ nuclear factor (erythroid-derived 2)-like 2 activator tert-butylhydroquinone (tBHQ; Sigma), or $50 \mu \mathrm{M}$ of the peroxisome proliferator-activated receptor $\alpha$ (PPAR $\alpha$ ) agonist Wy14,643 (Wy; 4-chloro-6(2,3-xylidino)-2-pyrimidinylthioacetic acid; gift from Dr. M. Schwarz, Tübingen, Germany). All compounds were dissolved in DMSO. Additional cell treatment was performed with $10 \mathrm{ng} / \mathrm{ml}$ of the inflammatory mediator IL-6 (PromoCell, Heidelberg, Germany), with equimolar mixtures of the fatty acids oleate and palmitate $(\mathrm{O} / \mathrm{P}$; dispersed in cell culture medium by sonication; both compounds purchased from Sigma), or with $10 \mu \mathrm{M}$ of the liver X receptor (LXR) agonist GW 3965 (Sigma). Oleate and palmitate were chosen as representatives of unsaturated and saturated fatty acids, respectively, and are the two fatty acids that show the highest abundance in NAFLD and NASH patients (Araya et al., 2004; please also refer to the Discussion section). For details on the treatment regimen, please also refer to the legend to Fig. 1.

The concentrations of receptor agonists were chosen based on initial cytotoxicity testing and respective literature values (Braeuning et al., 2015) to ensure robust and substantial activation of the respective receptor and transcription of its downstream targets in the absence of toxic injury. The selected concentration of IL-6 as a model inflammatory mediator $(10 \mathrm{ng} / \mathrm{ml})$ is

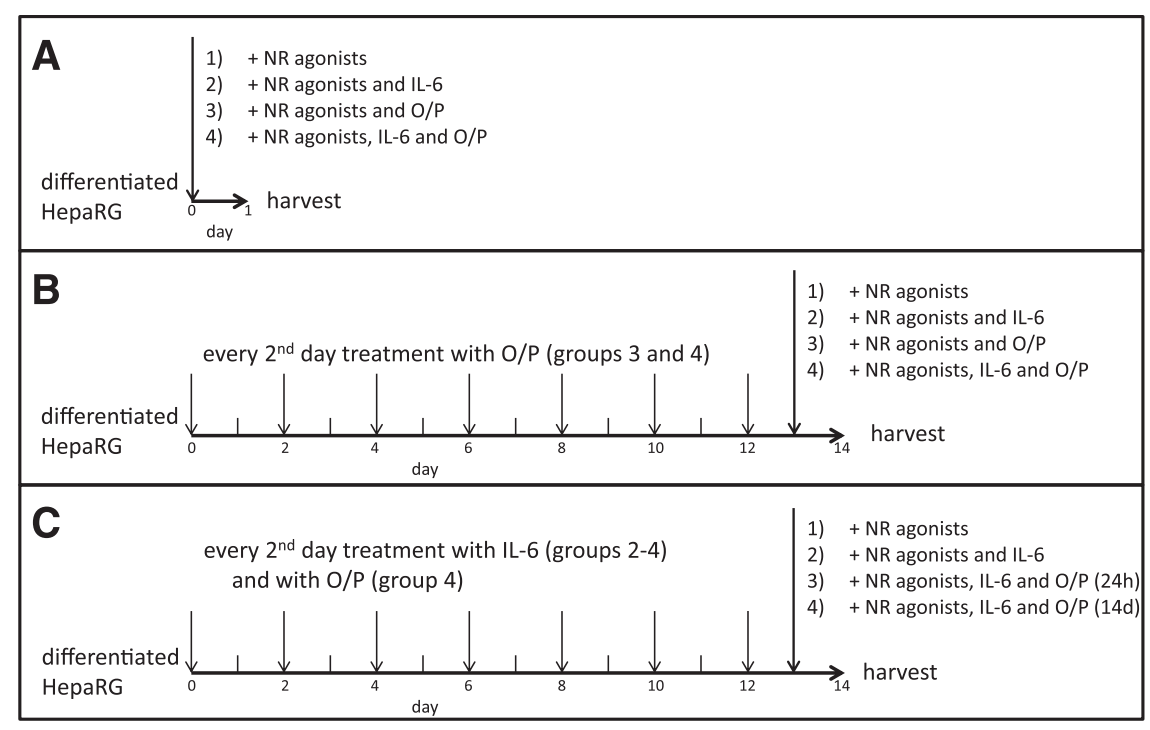

Fig. 1. Schematic setup of cell treatment. HepaRG cells were treated with different nuclear receptor agonists (or DMSO as a control) as detailed in the Materials and Methods section (5 $\mu \mathrm{M}$ 3-MC, $5 \mu \mathrm{M}$ CITCO, $10 \mu \mathrm{M}$ Rif, $30 \mu \mathrm{M}$ tBHQ, $50 \mu \mathrm{M}$ Wy), alone or in combination with $10 \mathrm{ng} / \mathrm{ml} \mathrm{IL}-6$ to mimic inflammatory conditions, and/or with $\mathrm{O} / \mathrm{P}(125 \mu \mathrm{M}$ each) to induce steatosis of hepatocytelike HepaRG cells. Different approaches were followed to analyze short- as well as long-term effects of treatment. (A) For analysis of short-term effects, differentiated HepaRG cells were incubated for 24 hours before harvest. (B) Longterm steatosis effects were analyzed using a treatment regimen based on 2 weeks of pretreatment with $\mathrm{O} / \mathrm{P}$ before 24 hours of treatment with nuclear receptor agonists and/or IL-6. (C) Long-term inflammatory effects were analyzed using a treatment regimen based on 2 weeks of pretreatment with IL-6, and long-term combined inflammatory and steatotic effects (mimicking steatohepatitis) were analyzed using a treatment regimen based on 2 weeks of pretreatment with IL-6 and O/P. 
substantially higher than IL- 6 serum levels in NASH patients $[\sim 10 \mathrm{pg} / \mathrm{ml}$ in NASH patients vs. $\sim 6 \mathrm{pg} / \mathrm{ml}$ in healthy controls; e.g., see Kugelmas et al. (2003)]. A supraphysiologic concentration, however, is needed to provoke the full response of HepaRG cells to IL-6 regarding the inhibitory effects on the transcription of selected P450 genes or induction of the acute-phase marker CRP (Klein et al., 2015; Rubin et al., 2015). The selected concentration of $10 \mathrm{ng} / \mathrm{ml}$ was tested for cytotoxicity to ensure the absence of nonspecific effects resulting from cell stress. The concentration of a 1:1 O/P mixture was based on initial cytotoxicity testing and pretests for cellular lipid accumulation.

Transfection and Luciferase Reporter Assay. Transfections were carried out in HepG2 human hepatoma cells for their low transfection efficiency in differentiated HepaRG cells. HepG2 cells were transfected using the method described in Luckert et al. (2015). For monitoring the activities of the nuclear receptors PPAR $\alpha$ and LXR, luciferase reporter systems based on fusion constructs of the GAL4 ligand binding domain with the ligand binding domain of the respective receptor were used as described in more detail by Luckert et al. (2015). The fusion constructs were encoded by pSG5-derived plasmids expressing the respective proteins under the control of the simian virus 40 (SV40) promoter. The activities of CAR and PXR were analyzed by use of a luciferase reporter driven by a fragment of the CAR- and PXR-responsive human CYP2B6 gene (Zukunft et al., 2005) in combination with cotransfected expression vectors for human CAR (pSG5-based, SV40 promoter-driven) or PXR (pCR3-based, CMV promoter-driven). Wild-type HepG2 cells are deficient in both previously mentioned receptors. For monitoring AHR activity, a pT81luc-based luciferase reporter system driven by an array of three AHR-binding dioxin response elements from the human CYP1A1 gene promoter was transfected (Schulthess et al., 2015). A plasmid encoding Renilla luciferase under the control of a constitutively active promoter was cotransfected for normalization purposes. Cells were treated 24 hours after transfection for 24 hours and then assayed for firefly and Renilla luciferase activities, as recently described (Luckert et al., 2015).

Cytotoxicity Testing. Cytotoxicity of IL-6 and equimolar mixtures of O/P was investigated in 96-well plates using the Cell Titer Blue (Promega, Mannheim, Germany) and MTT [3-(4,5-dimethylthiazole-2-yl)-2,5-diphenyltetrazoliumbromide] tests according to standard protocols. Triton X-100 (0.1\%) was used as a positive control. Only concentrations yielding cell viabilities $>80 \%$ were considered noncytotoxic and used for subsequent cell treatment.

Oil Red O Staining. Accumulation of fatty acids was monitored by staining with the dye Oil Red O (Sigma). Cells were fixed by $3.7 \%$ formaldehyde for 1 hour, followed by 1 hour of staining with a sterile-filtered $(0.45-\mu \mathrm{m}$ pore size), freshly prepared $0.18 \%$ Oil Red O solution in $84 \%$ isopropanol. Cells were washed with distilled water and with $60 \%$ isopropanol and then analyzed by light microscopy, or the cells were lysed in $0.7 \%$ sodium dodecyl sulfate in isopropanol for quantitative analysis of Oil Red $\mathrm{O}$ absorption at $\lambda=500 \mathrm{~nm}$ in a multiwell plate reader (Infinite M200 Pro; Tecan Group Ltd., Männedorf, Switzerland).

RNA Isolation and Gene Expression Analysis. Gene expression analysis was performed according to a previously published approach (Braeuning et al., 2015). Total RNA was isolated using the RNeasy Mini Kit (Qiagen, Hilden, Germany) according to the manufacturer's instructions and including an on-column genomic DNA digestion step with RNase-free DNase. Reverse transcription of RNA was carried out using TaqMan reverse transcription reagents (Applera GmbH, Darmstadt, Germany). A selection of 90 transcripts (Supplemental Table 1) related to functions in xenobiotic metabolism, fatty acid metabolism, and inflammation was quantified using a Fluidigm BioMark HD high-throughput quantitative chip platform (Fluidigm Corp., South San Francisco, CA), as recently described (Braeuning et al., 2015). Levels of mRNA expression were normalized to the expression of the reference gene glyceraldehyde-3-phosphate dehydrogenase $(G A P D H)$. Calculation of relative gene expression changes was done using the $\Delta \Delta \mathrm{Ct}$ method.

Verification of selected gene expression changes was performed for the cell culture conditions outlined in Fig. 1, A and B, by conventional SYBR Green I-based real-time reverse transcription-polymerase chain reaction (RT-PCR) with the Maxima SYBR Green/ROX qPCR Mastermix (Thermo Fisher Scientific, Berlin, Germany) on a LightCycler 96 machine (Roche, Mannheim, Germany) after reverse transcription with the help of the high-capacity cDNA reverse transcription kit (Applied Biosystems, Darmstadt, Germany) using the $\Delta \Delta \mathrm{Ct}$ quantification method with $G A P D H$ as a reference gene, for which no systematic treatment effects were recorded. The following primers were used for RT-PCR analysis: $C R P$ fwd 5'-GGCGAAGTGTTCACCAAACC-3', CRP rev 5'-AAAGCGGGAGGTACCAGAGA-3'; CYP2B6 fwd 5'-TTCCTACTGCTTCCGTCTATCAAA-3', CYP2B6 rev
5'-GTGCAGAATCCCACAGCTCA-3'; CYP3A4 fwd 5'-TCAGCCTGGTGCTCCTCTATCTAT-3', CYP3A4 rev 5'-AAGCCCTTATGGTAGGACAAAATATTT-3'; FABPI fwd 5'-CAAGTTCACCATCACCGCTGGGTC-3', FABP1 rev 5' -TCATTGTCTCCAGCTCACATTCCTC-3'; GAPDH fwd 5'-ATTTGGCTACAGCAACAGGG-3', GAPDH rev 5'-CAACTGTGAGGAGGGGAGA-3'.

P450 Activity Assays. Determination of P450 activities was performed using a multiplex liquid chromatography-tandem mass spectrometry (MS) detection method of metabolic conversion of model P450 substrates. Analyses were carried out according to Luckert et al. (2017) with minor modifications as follows: cells were incubated for 2 hours with a substrate mix containing model P450 substrates [50 $\mu \mathrm{M}$ phenacetin (CYP1A2), $25 \mu \mathrm{M}$ bupropion (CYP2B6), $5 \mu \mathrm{M}$ amodiaquine (CYP2C8), $100 \mu \mathrm{M}$ tolbutamide (CYP2C9), $100 \mu \mathrm{M}$ S-mephenytoin (CYP2C19), $5 \mu \mathrm{M}$ propafenone (CYP2D6), and $10 \mu \mathrm{M}$ midazolam (CYP3A4)]. Cell culture supernatant samples were mixed with $10 \%$ of $250 \mathrm{mM}$ formic acid, immediately frozen, and then used for MS-based analysis of metabolites according to Luckert et al. (2017) and references therein. Data were normalized to cell viability values.

Statistical Analysis. Gene expression, reporter assay, and P450 activity data were analyzed using SigmaPlot software (version 13). For comparing the means between different groups, we used the parametric one-way analysis of variance (ANOVA) test assuming normal distribution of data. ANOVA is insensitive to deviations from normal distribution and thus can also be used if the criteria of normality are not strictly met for all data (McDonald, 2014). Furthermore, parametric tests are preferred for parametric data as they are more powerful than nonparametric tests in which the original parametric data must be first converted to ranks, leading to a loss of information and resulting in a loss of power. If statistically significant differences between treatment groups were detected by ANOVA analyses, the Bonferroni post hoc test was subsequently used to identify which treatment groups differ from the control group. For P450 activity and gene expression data, statistical analysis was independently performed for treatment scenario 1 (only NR agonists), scenario 2 (NR agonists and IL-6), scenario 3 (NR agonists and O/P), and scenario 4 (NR agonists, Il-6, and O/P) within the treatment schemes A, B, and C (see Fig. 1) and the five treatments with the different NR agonists were compared with the solvent control group. Additionally, the solvent control groups of treatment scenarios 2, 3, and 4 were compared with solvent control group of treatment scenario 1 to analyze the effect of IL-6, O/P, or a combination of both IL- 6 and O/P in the absence of NR agonists. For reporter gene assay data, statistical analysis was independently performed for the noninduced and the induced nuclear receptors and the three treatment groups (Il-6, O/P, and a combination of both IL- 6 and O/P) were compared with solvent control group. $P$ value $<0.05$ was considered significant for ANOVA and post hoc analysis.

\section{Results}

In initial experiments, cell viability testing revealed no indication of cytotoxic effects of the nuclear receptor agonists, the 1:1 mixture of $\mathrm{O} / \mathrm{P}$, and IL-6 as inflammatory mediator (Supplemental Fig. 1). It was important to exclude unspecific effects in subsequent experiments caused by cell stress. Dose-dependent induction of steatosis by $\mathrm{O} / \mathrm{P}$ treatment was verified by triglyceride staining of HepaRG cells using Oil Red O (Supplemental Fig. 2).

To allow for a comprehensive analysis of the interplay of nuclear receptor-mediated induction of drug-metabolizing enzymes, steatosis, and inflammatory signaling, HepaRG human hepatocarcinoma cells were treated according to the scheme depicted in Fig. 1. The experimental setup comprised both short-term (24 hours) as well as long-term (14 days) treatments with the fatty acids and/or the inflammatory mediator. We thus aimed to mimic three different conditions: 1) acute, short-term fatty acid overflow and/or inflammation; 2) conditions of prolonged fatty acid overflow resulting in steatosis as observed in early fatty liver disease; and 3) conditions of steatotic liver cells in an inflammatory environment as observed in steatohepatitis patients.

Effects on Inflammation- and Fat Metabolism-Related Genes. In total, expression of a panel of 91 genes was measured, resulting in $>20,000$ individual data points. The gene set was selected based on 
literature data and consisted of genes related to hepatic drug metabolism, fatty acid metabolism, and inflammation. These data are summarized in Supplemental Table 1. Treatment with O/P barely affected the transcription of inflammation-related genes (Supplemental Fig. 3). Short-term as well as long-term treatment with IL-6 strongly induced the transcription of the model inflammation marker c-reactive protein (CRP), an acute-phase protein and frequently used marker for NASH in vivo (Fierbinteanu-Braticevici et al., 2011). Similarly, the mRNA for serum amyloid A1/2, another acute-phase protein, was consistently upregulated by IL-6, thus confirming that IL- 6 had in fact exerted the desired biologic effects in HepaRG cells (Supplemental Fig. 3). Although no effects on the mRNAs encoding the cytokines CCL20, IL-6, and IL- 8 were observed with 24 hours of IL-6 treatment, these transcripts were upregulated after long-term incubation with IL-6 for 14 days, together with an upregulation of the mRNA encoding PTGS, an enzyme engaged in prostaglandin biosynthesis (Supplemental Fig. 3). Of the nuclear receptor agonists, only the AHR activator 3-MC influenced numerous inflammation-related genes to a remarkable degree (Supplemental Fig. 3).

When inspecting alterations in the expression of fat metabolismrelated genes, it was observed that IL- 6 treatment led to a downregulation of most of these genes (Supplemental Fig. 4). The presence of O/P for 24 hours or 14 days, as well as incubation of HepaRG cells with the different nuclear receptor agonists, did not substantially affect fat metabolism-related gene expression, except 3-MC, which downregulated a number of relevant mRNAs (Supplemental Fig. 4); however, these alterations by IL-6 and 3-MC were not accompanied by remarkable changes in steatosis induction by $\mathrm{O} / \mathrm{P}$ since Oil Red $\mathrm{O}$ staining revealed unchanged steatosis induction by $\mathrm{O} / \mathrm{P}$ in the presence of IL-6 and nuclear receptor agonists, after both 24 hours and 14 days (data not shown).

Effect of Inflammatory Conditions and Fat Overload on P450 Expression and Activities. Data for the 12 different P450 isoforms contained in the expression analysis are presented in Fig. 2 (for additional non-P450 genes involved in phase 1 of drug metabolism, see Supplemental Fig. 5). Treatment with IL-6 for 24 hours or 14 days led to pronounced downregulation of basal expression [i.e., in solvent (DMSO)-treated cells when xenobiotic activators of nuclear receptors were absent] of most P450 isoforms (Fig. 2, two left panels), an effect that was especially pronounced after repeated cell treatment over a period of 2 weeks (Fig. 2C; see also Supplemental Table 1 for underlying data). Treatment with $\mathrm{O} / \mathrm{P}$ for 24 hours or 14 days exerted only minor effects, with a tendency for downregulation of some P450 isoforms (Fig. 2, A and B, third panel from left). Alterations in P450 expression after combined treatment with O/P and IL- 6 for 24 hours (Fig. 2A, right panel) or 2 weeks (Fig. 2C, right panel) or after 24 hours of IL-6 treatment after 13 days of pretreatment with $\mathrm{O} / \mathrm{P}$ (Fig. 2B, right panel) were similar to corresponding alterations monitored with IL-6 treatment alone. Downregulation of P450s was confirmed by analyses of the enzymatic activities of seven different $\mathrm{P} 450 \mathrm{~s}$, which revealed a pronounced effect on drug metabolism-related P450 activities by IL-6 and also slight downregulation of basal P4540 enzyme activities in steatotic cells after 14 days of O/P treatment (Fig. 3). For detailed data underlying the latter figure, please refer to Supplemental Table 2.

Inflammatory Conditions Diminish Xenobiotic Induction of CAR- and PXR-, but not of AHR-Dependent P450 Isoforms. The presence of xenobiotic activators of ligand-activated receptors in HepaRG cells not treated with O/P or IL-6 led to the expected induction of target genes of the respective receptors, with comparable patterns of P450 induction after 24 hours or 14 days of cultures (Fig. 2, left panels). Activation of the mRNAs encoding the AHR-dependent $\mathrm{P} 450$ isoforms CYP1A1 and CYP1A2 by 3 -MC was striking; CITCO induced the expression of the CAR target genes $C Y P 1 A 2, C Y P 2 B 6$, and $C Y P 3 A 4$, whereas the PXR activator rifampicin (Rif) induced $C Y P 2 B 6$ and CYP3A4 mRNAs (Fig. 2). The latter P450 isoform was also induced when cells were treated with the PPAR $\alpha$ agonist WY14,643, whereas the nuclear factor (erythroid-derived 2)-like 2 activator tBHQ did not exert strong effects on P450 expression (Fig. 2). Treatment with IL-6, again visible more pronouncedly after 2 weeks, efficiently counteracted the inductive effects of nuclear receptor agonists, except 3-MCmediated induction of $C Y P 1 A 1 / C Y P 1 A 2$, which remained rather stable under these conditions (Fig. 2). Short-term treatment with O/P exerted only minor effects on nuclear receptor-mediated $\mathrm{P} 450$ induction, as evidenced by a very similar $\mathrm{P} 450$ induction pattern in O/P-treated cells and solvent controls (Fig. 2A). As for basal P450 expression, data on xenobiotic-induced transcription of selected P450 isoforms also indicated a broad-spectrum inhibitory effect of IL-6 treatment, especially on CAR and PXR target P450s, which was most obvious after 2 weeks of repeated IL-6 treatment (Fig. 2); however, an interesting finding was that 3-MC-, and thus AHR-mediated induction of CYP1A1 and CYP1A2 mRNAs, was not remarkably diminished after incubations of cells in the presence of IL-6 (Fig. 2).

The inhibition of CAR- and PXR-dependent xenobiotic-induced P450 enzyme activities by IL-6 was confirmed by MS analyses (Fig. 3; Supplemental Table 2); and, in line with the results from the mRNA expression analyses, only the induction of the AHR-dependent enzyme CYP1A2 by 3-MC was exempt from the repressive action of IL- 6 on the P450s (Fig. 3). Of note, elevated CYP2D6 enzyme activity levels were measured in the supernatants of 3-MC-treated cells. This finding, however, should be interpreted with care because overall CYP2D6 activities in the HepaRG cell lines are extremely low.

The non-IL-6- and non-O/P-treated cells in Fig. 2, B and C stem from independent experiments performed under identical conditions (i.e., cultivation of the HepaRG cells in a differentiated stage for 14 days, followed by treatment with nuclear receptor agonists for the last 24 hours of cultivation). Presented data show quite similar patterns of P450 induction at both the mRNA and enzyme activity levels (Fig. 2, B and C; Fig. 3, B and C), which demonstrates the high reproducibility and validity of the data set. In addition, selected data on xenobiotic- and IL-6-mediated alterations in gene expression (CYP2B6, CYP3A4, CRP, $F A B P 1)$ were independently verified using a standard real-time RT-PCR system. Data were well in agreement with the Fluidigm gene expression analysis results (data not shown).

Impact of O/P and IL-6 on mRNA Expression Related to Phase 2 and 3. Expression of a broad spectrum of mRNAs encoding proteins involved in phase 2 and phase 3 of xenobiotic metabolism was also analyzed (Supplemental Fig. 6). Similar to observations with P450 expression, the inflammatory mediator IL-6 reduced the expression of most of the genes analyzed (Supplemental Fig. 6). O/P treatment for 24 hours did not exert remarkable effects, whereas a slight tendency for downregulation of some genes was observed after 14 days of incubation in the presence of O/P (Supplemental Fig. 6). Induction of phase 2/phase 3-related genes by xenobiotics was, as expected, much less pronounced than for most P450 genes, and as observed with phase 1 enzymes, xenobiotic effects on phase 2/phase 3-related gene transcription were also dampened by IL-6 (Supplemental Fig. 6). As an exception, SULT1B1 mRNA levels were increased by IL-6 after 14 days of treatment, but not after 24 hours of IL-6 treatment (Supplemental Fig. 6).

Impact of O/P and IL-6 on Xenobiotic-Activated Transcription Factor Expression and Activity. A selection of nuclear receptors and transcription factors engaged in the regulation of drug-metabolizing enzymes was analyzed at the mRNA level in response to the different treatments (Fig. 4). Generally, the effects of exogenous agonists and O/P treatment were not prominent, except for AHR and vitamin D receptor 

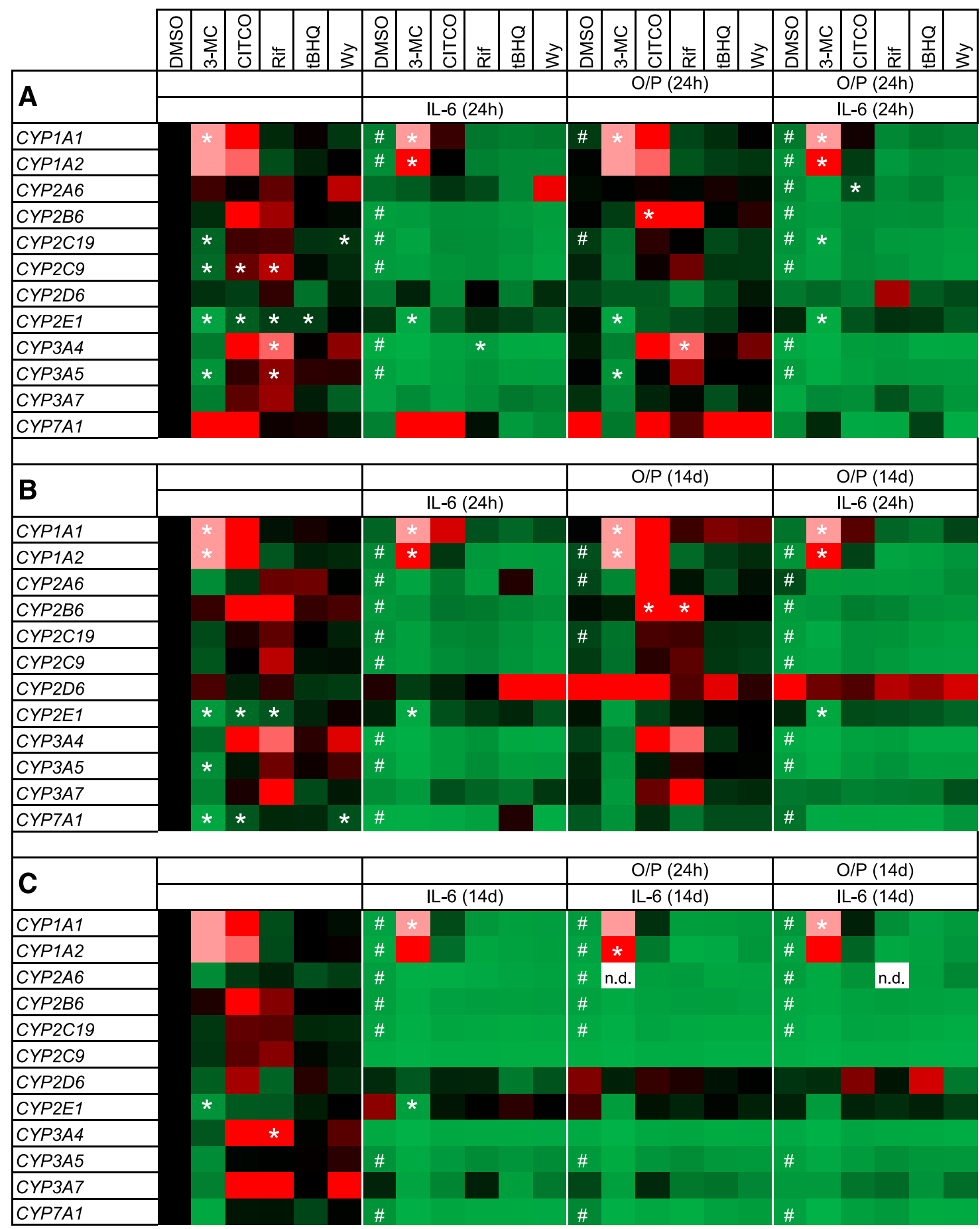

0.5

1

1.5

$2 \quad 5 \quad 10 \quad 20$

Fig. 2. Heat-map visualization of the expression of P450 genes in HepaRG cells and their regulation by nuclear receptor agonists $(5 \mu \mathrm{M} 3-\mathrm{MC}, 5 \mu \mathrm{M}$ CITCO, $10 \mu \mathrm{M}$ Rif, $30 \mu \mathrm{M} \mathrm{tBHQ}, 50 \mu \mathrm{M} \mathrm{Wy}), \mathrm{O} / \mathrm{P}(125 \mu \mathrm{M}$ each), and IL-6 $(10 \mathrm{ng} / \mathrm{ml})$. Cells were treated according to the scheme presented in Fig. 1, and mRNA expression levels were analyzed using a Fluidigm PCR system (Fluidigm Corp.). Differentiated HepaRG cells were either incubated with the test compounds for 24 hours before harvest (A) or using a treatment regimen based on 2 weeks of pretreatment with O/P before 24 hours of treatment with nuclear receptor agonists and/or IL-6 (B) or using a treatment regimen based on 2 weeks of pretreatment with IL-6 and/or IL-6 and O/P (C). Mean relative values ( $n=3$ independent biologic replicates) are given. *Statistical significance in comparison with solvent control $(P<0.05)$; " statistical significance compared with solvent control of cells not treated with IL-6 and/or O/P.

upregulation by the AHR agonist 3-MC, coupled to a downregulation of other nuclear receptors such as CAR and PXR (Fig. 4). IL-6 resulted in downregulation of most genes of this category, including the important xeno-sensors CAR and PXR. The latter effect was consistently observed with short- as well as long-term IL-6 treatment (Fig. 4). Downregulation of AHR mRNA was statistically significant after 24 hours of IL-6 


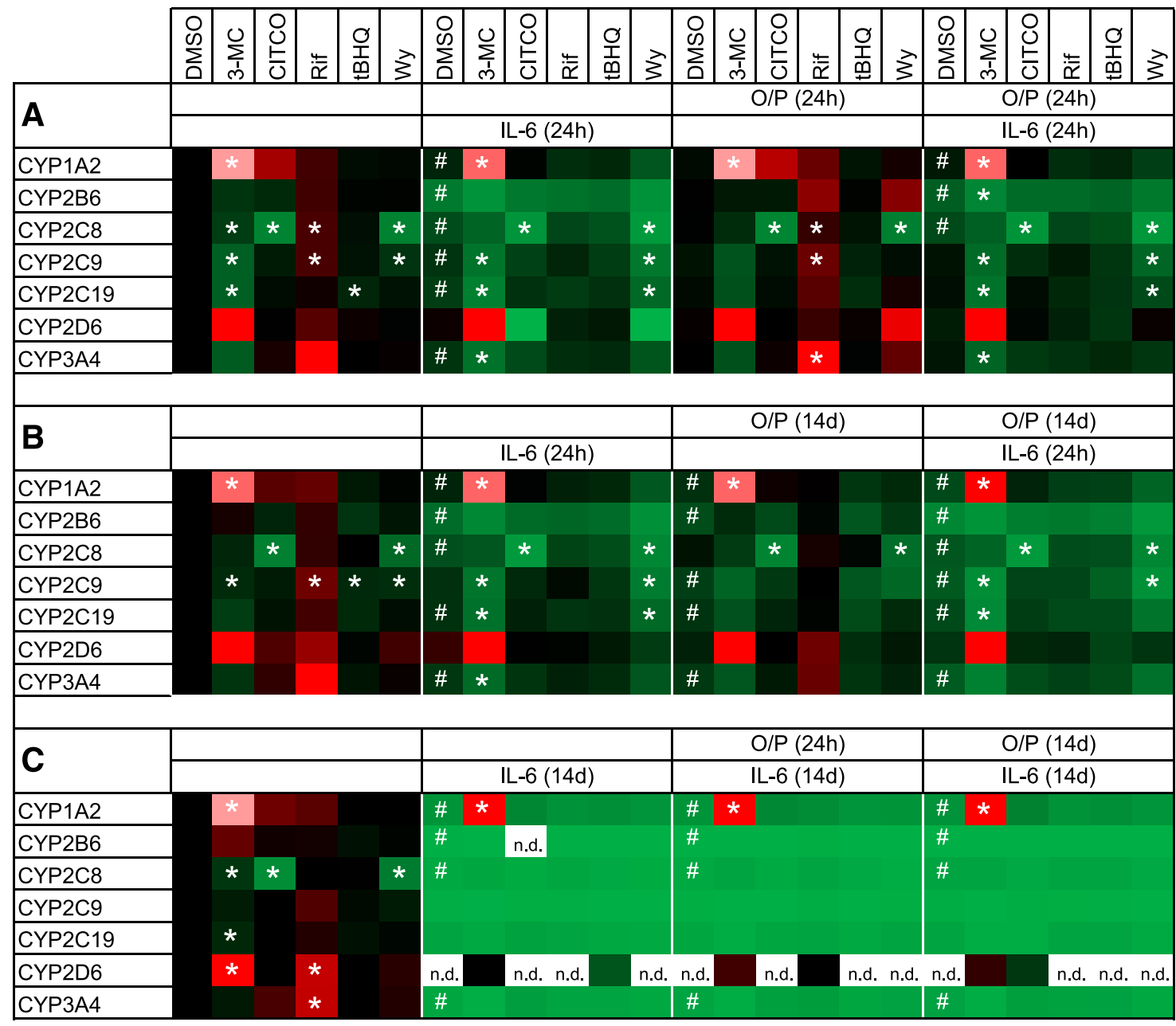

fold activity

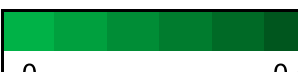

0.5

1.5

$\begin{array}{llll}2 & 5 & 10 & 20\end{array}$

Fig. 3. Heat-map visualization of the activities of P450 enzymes in HepaRG cells and their regulation by nuclear receptor agonists $(5 \mu \mathrm{M} 3-\mathrm{MC}, 5 \mu \mathrm{M}$ CITCO, $10 \mu \mathrm{M}$ Rif, $30 \mu \mathrm{M} \mathrm{tBHQ}, 50 \mu \mathrm{M}$ Wy), O/P (125 $\mu \mathrm{M}$ each), and IL-6 (10 ng/ml). Cells were treated according to the scheme presented in Fig. 1, and P450 activities were assayed using model substrate compounds and liquid chromatography-mass spectroscopy-based analytics. Differentiated HepaRG cells were either incubated with the test compounds for 24 hours before harvest (A) or using a treatment regimen based on 2 weeks of pretreatment with O/P before 24 hours of treatment with nuclear receptor agonists and/or IL-6 (B), or using a treatment regimen based on 2 weeks of pretreatment with IL- 6 and/or IL-6 and O/P (C). Mean relative values $(n=3$ independent biologic replicates) are given. *Statistical significance in comparison with solvent control $(P<0.05)$; ${ }^{*}$ statistical significance compared with solvent control of cells not treated with IL-6 and/or O/P. For raw data on P450 activities given in picomoles per milliliter of cell culture supernatant, please refer to Supplemental Table 2.

treatment but failed our criteria of statistical significance in the long-term experiments (Fig. 4).

In principle, downregulation of nuclear receptor target genes (e.g., the P450s by IL-6) might be a consequence of diminished availability of the nuclear receptors themselves or due to an interference of IL-6dependent signaling with the transactivation potential of the receptors. To follow up on the findings of the impact of IL-6 treatment on nuclear receptorencoding mRNAs, as well as on nuclear receptor target genes, the human hepatoma cell line HepG2 was chosen for subsequent reporter gene analyses of the transcriptional activity of nuclear receptors under IL-6 and $\mathrm{O} / \mathrm{P}$ treatment because HepaRG cells showed poor transfection efficiency with different plasmids. Basal as well as ligand-induced activities of receptors AHR, CAR, PXR, PPAR $\alpha$, and LXR were assessed using luciferase-based assays. In these assays, physiologic effects of IL- 6 on nuclear receptor mRNA expression were eliminated by using appropriate vectors expressing the receptors (native or in the form of GAL4-based fusion proteins) under control of the constitutively active cytomegaly virus or SV40 promoters. As presented in Fig. 5A, inspection of luciferase reporter data on basal (i.e., in the absence of xenobiotic inducers) nuclear receptor activities revealed that the transactivation potential of the nuclear receptors CAR, PXR, and LXR was significantly inhibited by IL-6. O/P treatment also weakly inhibited basal CAR activity, whereas a remarkable induction of LXR and $\operatorname{PPAR} \alpha$ activities was recorded after treatment with $\mathrm{O} / \mathrm{P}$ (Fig. 5A). None of the treatment protocols exerted pronounced effects on the AHR (Fig. 5A). For the xenobiotic-regulated receptors AHR, CAR, PXR, and PPAR $\alpha$, the assays were repeated in the presence of strong model activators of the respective receptors (Fig. 5B). Treatment of HepG2 cells with $\mathrm{O} / \mathrm{P}$ barely affected xenobioticinduced reporter activities driven by these receptors (Fig. 5B). By contrast, IL-6 substantially inhibited the activities of the CAR-, PXR-, and PPAR $\alpha$-dependent reporter systems, but it did not affect 3-MC-induced AHR activity at the CYP1A1 promoter-derived 3 dioxin reporter element (Fig. 5B). 

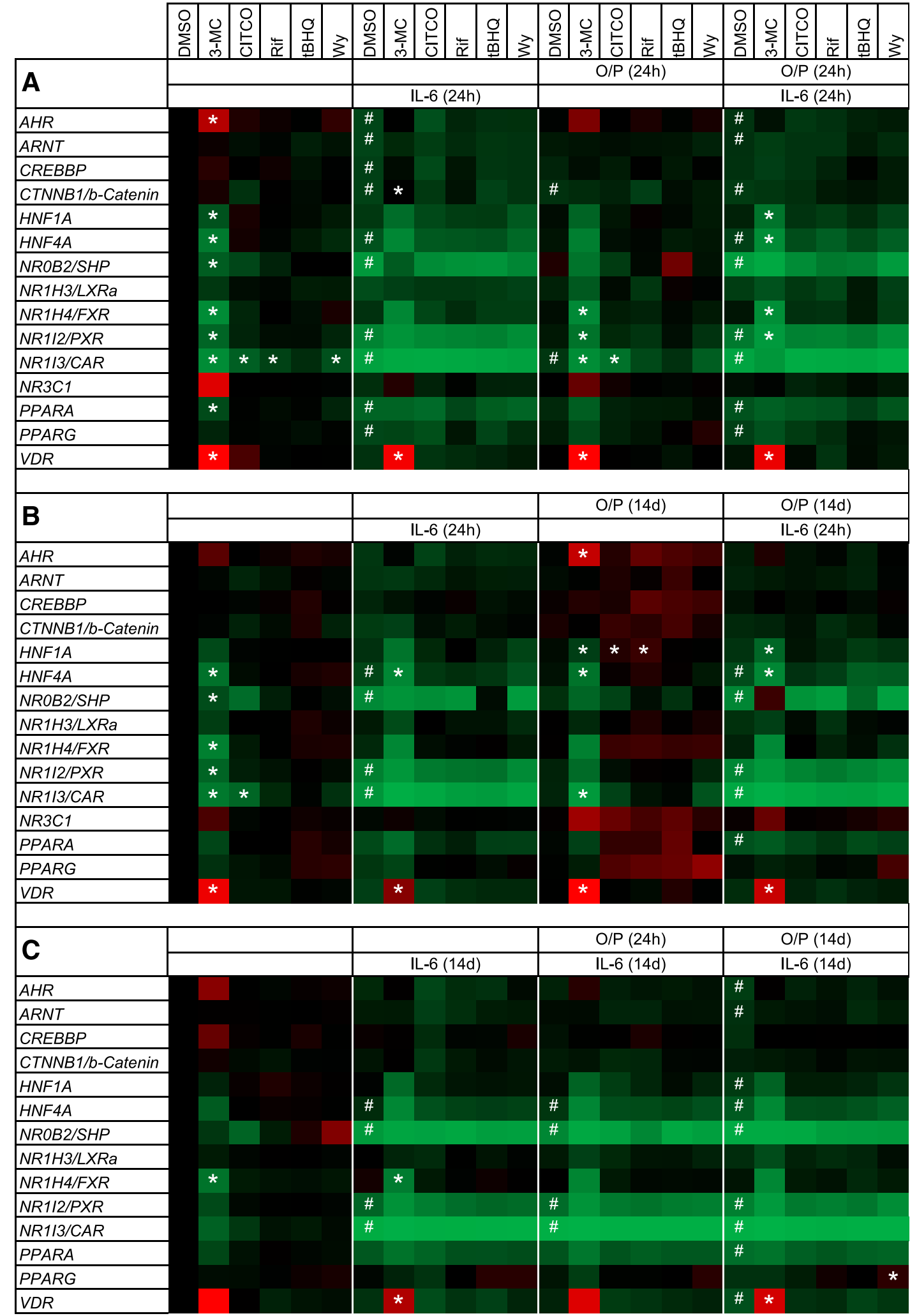

fold expression

0.5

1.5

\begin{tabular}{llll}
2 & 5 & 10 & 20 \\
\hline
\end{tabular}

Fig. 4. Heat-map visualization of the expression of nuclear receptors genes and genes encoding other transcriptional regulators with relevance for the regulation of xenobiotic metabolism in HepaRG cells. Their regulation by nuclear receptor agonists ( $5 \mu \mathrm{M}$ 3-MC, $5 \mu \mathrm{M}$ CITCO, $10 \mu \mathrm{M}$ Rif, $30 \mu \mathrm{M}$ tBHQ, $50 \mu \mathrm{M} \mathrm{Wy}), \mathrm{O} / \mathrm{P}(125 \mu \mathrm{M}$ each), and IL-6 (10 ng/ml). after treatment of cells according to the scheme presented in Fig. 1 is depicted. mRNA expression levels were analyzed using a Fluidigm PCR system (Fludigm Corp.). Differentiated HepaRG cells were either incubated with the test compounds for 24 hours before harvest (A) or using a treatment regimen based on 2 weeks of pretreatment with O/P before 24 hours of treatment with nuclear receptor agonists and/or IL-6 (B) or using a treatment regimen based on 2 weeks of pretreatment with IL-6 and/or IL-6 and O/P (C). Mean relative values $(n=3$ independent biologic replicates) are given. *Statistical significance in comparison with solvent control $(P<0.05)$; \#indicate statistical significance compared with solvent control of cells not treated with IL-6 and/or O/P. 

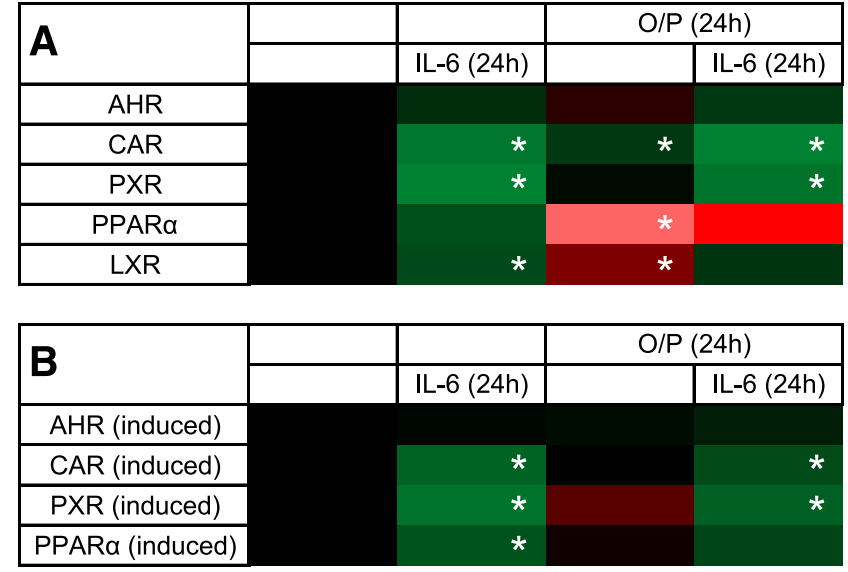

fold activity

\begin{tabular}{|llllllll|}
\hline 0 & 0.25 & 0.5 & 0.75 & 1 & 1.5 & 3 & 7 \\
\hline
\end{tabular}

Fig. 5. Heat-map visualization of the transcriptional activities of nuclear receptors engaged in the regulation of drug and lipid metabolism, as determined by luciferase reporter analyses in HepG2 cells. Regulation of the basal activities of AHR, CAR, PXR, LXR, and PPAR $\alpha$ by O/P (125 $\mu \mathrm{M}$ each) and IL-6 $(10 \mathrm{ng} / \mathrm{ml})$. in the absence of xenobiotic receptor activation (A), as well as regulation of xenobiotic-induced activities of AHR (5 $\mu \mathrm{M} 3-\mathrm{MC})$, CAR $(5 \mu \mathrm{M}$ CITCO), PXR (10 $\mu \mathrm{M}$ Rif), and PPAR $\alpha(50 \mu \mathrm{M}$ Wy) by O/P (125 $\mu \mathrm{M}$ each) and IL-6 $(10 \mathrm{ng} / \mathrm{ml})(\mathrm{B})$ is shown. Mean values $(n=6-9$ independent biologic replicates; each in two to three technical replicates) are given relative to solvent controls (A) or receptor agonists (B); asterisks indicate statistical significance in comparison with solvent control $(P<0.05)$. Treatment with receptor agonists led to a mean induction of the respective reporter signals by the following

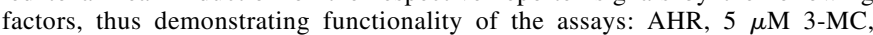
50-fold; CAR, $5 \mu \mathrm{M}$ CITCO, 2.8-fold; PXR, $10 \mu \mathrm{M}$ Rif, 3.1-fold; PPAR $\alpha$, $50 \mu \mathrm{M}$ Wy, 103-fold; LXR, $10 \mu \mathrm{M}$ GW3965, 472-fold.

\section{Discussion}

The present study provides novel insights into the effects of fatty acid accumulation and/or inflammatory signals on drug metabolism in HepaRG cells. This work thereby confirms, extends, and complements earlier investigations on the effects of fatty acid accumulation on the regulation of drug-metabolizing enzymes in human primary hepatocytes, HepaRG cells, or rodent cells by inflammatory mediators (AbdelRazzak et al., 1993; Abdel-Razzak et al., 1995; Pascussi et al., 2000; Klein et al., 2015; Rubin et al., 2015). In extension of the aforementioned studies, which focused on the influence of inflammation on drug metabolism in its basal (this means in the absence of xenobiotic inducers of drug metabolism) state and under short-term treatment conditions, the present study was done to elucidate the role of interactions between steatosis, inflammation, and nuclear receptors on drug metabolism and its inducibility.

The results underline the suitability of HepaRG cells as an in vitro model of hepatic steatosis. This cell line has previously been used for steatosis induction with an O/P mixture (Brown et al., 2013) or with oleic acid alone (Antherieu et al., 2011; Rogue et al., 2014). Oleate and palmitate constitute the most abundant fatty acids in NAFLD and NASH patients, and the relative composition of liver fatty acids significantly shifts toward oleate and palmitate when NAFLD or NASH livers are compared with healthy controls (Araya et al., 2004). Thus, the chosen way of inducing steatosis in vitro by an overload with these two fatty acids is considered in line with the physiologic development of steatosis in vivo. In general, and compared with the effects of IL-6 or nuclear receptor agonists, transcriptional alterations induced by $\mathrm{O} / \mathrm{P}$ were rather moderate. Despite pronounced accumulation of fatty acids, as measured by Oil Red $\mathrm{O}$ staining, only a few transcriptional responses were recorded after 24 hours of O/P treatment. Thus, long-term cultivation and treatment might be more suited for in vitro simulation of NAFLD than a short-term fatty acid overload approach. O/P did not substantially affect basal and xenobiotic-induced activities of AHR, CAR, and PXR and also of their target mRNAs, in contrast to PPAR $\alpha$ and LXR. Receptor activation by xenobiotics also did not significantly influence fatty acid accumulation in our own experiments, in which we used HepaRG cells under cell culture conditions identical to the experimental setup from this study (see Fig. 1, all conditions from panels A and B) in combination with Oil Red $\mathrm{O}$ staining as a readout for fatty acid accumulation (our own unpublished data). This lack of impact of xenobiotic receptor agonists on steatosis of HepaRG cells might appear to contrast with /previous results from Rogue et al. (2014), who have shown that $\operatorname{PPAR} \alpha$ activation in HepaRG cells can reduce liver steatosis; however, also in that study, no effects of $\operatorname{PPAR} \alpha$ agonists on steatosis were observed when the cells were treated with nuclear receptor agonists for only 24 hours. Thus, our observations are well in agreement with the data from Rogue et al. (2014) and support the hypothesis that only long-term presence of a $\operatorname{PPAR} \alpha$ activator can substantially affect fatty accumulation in HepaRG cells. In another study with HepG2 cells, linoleic acid weakly induced a CAR-driven luciferase reporter system (Finn et al., 2009), whereas a tendency for downregulation was observed with $\mathrm{O} / \mathrm{P}$ as fatty acids in our analyses. Differences between the fatty acids used for cell treatment (linoleic acid vs. equimolar mixture of $\mathrm{O} / \mathrm{P})$, as well as differences between the concentrations used [up to $12.5 \mu \mathrm{M}$ in Finn et al. (2009)] vs. $125 \mu \mathrm{M}$ each of $\mathrm{O}$ and $\mathrm{P}$ in this work) might explain the discrepancies in the results between the two studies.

Previous data have suggested a generally downregulating effect of IL-6 treatment on different players in drug metabolism, including metabolic enzymes at the mRNA and activity levels, as well as mRNAs encoding important nuclear receptors (Pascussi et al., 2000; Klein et al., 2015; Rubin et al., 2015) or hepatic transcription factors (Merrell and Cherrington, 2011; Liu et al., 2012; Klein et al., 2015) in primary human hepatocytes and/or HepaRG cells. Our own unpublished data similarly show that IL-6 downregulates many drug-metabolizing enzymes in HC-AFW1 human hepatocarcinoma cells, another well suited in vitro model for studying the regulation of human drug metabolism (Braeuning et al., 2015; Petzuch et al., 2015). The present data corroborate the findings on IL-6-mediated inhibition of drug metabolism and add the important aspect of long-term IL-6 treatment, which exerted much more pronounced effects on drug metabolism in HepaRG cells than did shortterm IL-6 treatment. Thus, repeated treatment with IL- 6 might be considered the superior HepaRG in vitro model for representing NASH compared with shorter treatments; however, it should be noted that, despite a strong and consistent response of HepaRG cells to IL-6 treatment observed with regard to the induction of cytokine production and acute-phase markers of inflammation, no substantial evidence for an IL-6-dependent induction of an oxidative stress response (such as, e.g., HMOX-1 or NQO1 gene transcription) has been recorded in the course of our analyses. Potentially, the longterm HepaRG inflammation model might thus further be optimized by developing it into a coculture model with immune cells, which might enhance reactive oxygen species formation and resulting stress to hepatocytes.

Previous data from the literature on the effect of IL-6 treatment on nuclear receptors are not entirely consistent; whereas observations on reduced cytochrome $\mathrm{P} 450$ activities indirectly suggest an involvement of nuclear receptors (Rubin et al., 2015), their downregulation at the mRNA level has been convincingly shown (Pascussi et al., 2000; Klein et al., 2015). Here we confirm nuclear receptor downregulation by IL-6 
and furthermore provide new insight by analyzing the effects of IL- 6 on the transcriptional activities of the nuclear receptors AHR, CAR, PXR, PPAR $\alpha$, and LXR. Interestingly, it appears that xenobiotic-induced gene expression via the AHR is not substantially affected by IL-6, whereas the other xeno-sensing receptors studied displayed remarkable inhibition. In contrast to our analyses, no effects on CAR-dependent reporter gene transactivation have been observed in a previous study (Pascussi et al., 2000). One might speculate that the response of HUH-7 cells used in the study by Pascussi et al. (2000) to IL-6 might not correspond to effects consistently observed in primary human hepatocytes, HepaRG cells, and HepG2 cells at the mRNA, enzyme activity, and reporter assay levels. The inhibition of nuclear receptor mRNA transcription described here and by others (Pascussi et al., 2000; Klein et al., 2015) might be connected to lower levels of the mRNAs or activities of HNF family members (Merrell and Cherrington, 2011; Liu et al., 2012; Klein et al., 2015). This hypothesis is consistent with the findings of the present study; however, it appears unlikely that the observed moderate loss of nuclear receptor mRNAs is solely responsible for decreased P450 expression and especially diminished xenobiotic-stimulated P450 induction in the presence of IL-6: nuclear receptors (or their GAL4 fusion construct variants) used for the reporter assays in this study were expressed from constitutively active promoters and have nonetheless revealed pronounced inhibition of reporter gene transactivation by IL-6, especially for CAR and PXR. Instead, the findings from the reporter gene analyses suggest that inhibition of the transactivation potential of certain nuclear receptors, rather than reduced nuclear receptor-encoding mRNA levels, is causative for the observed effects. This applies to CAR, PXR, PPAR $\alpha$, and LXR, but not to the AHR, the only receptor not substantially affected by IL- 6 treatment in our analyses. Except for the bHLH/PAS family protein AHR, which uses aryl hydrocarbon receptor nuclear translocator as its dimerization partner, the other investigated receptors dimerize with their common partner $\mathrm{RXR} \alpha$. Using an in silico modeling approach based on transcriptomic and phosphoproteomic data, it has recently been proposed that $\operatorname{RXR} \alpha$ plays a decisive role in mediating the downregulation of hepatic detoxification under inflammatory conditions (Keller et al., 2016). The present experimental data fit well into these model predictions by demonstrating that the transcriptional activity of different nuclear receptors using $\operatorname{RXR} \alpha$ is diminished when hepatic cells are treated with the inflammatory mediator IL-6, whereas the activity of the AHR, which acts independent of $\operatorname{RXR} \alpha$, is not affected. The exact mechanism of action by which IL-6 affects $\operatorname{RXR} \alpha$ remains to be elucidated.

Taken together, the present data provide a comprehensive overview of the influence of NAFLD- and NASH-like conditions on hepatic drug metabolism and its regulation by xenobiotics in human HepaRG cells in vitro. The cell system is well suited for this type of analysis, particularly when long-term effects are included in the focus of the study. According to the present data, it might be more appropriate to study liver disease conditions in vitro in long-term culture than by using single short-term incubation of the cells with the various effectors. Although demonstrating that fatty acid accumulation alone does not substantially influence the drug-metabolizing capacity of these liver cells, our results demonstrate massive inhibition of basal drug metabolism by the inflammatory mediator IL- 6 and show that xenobioticinduced levels of drug-metabolizing enzymes regulated by CAR and PXR, but not AHR, are similarly influenced by the cytokine.

\section{Acknowledgments}

We thank Dr. M. Schwarz (Tübingen, Germany) for the gift of Wy14,643, Silvia Vetter (Tübingen, Germany) for supporting cell culture experiments, Ute Hofmann and Markus König (Stuttgart, Germany) for mass spectrometric analyses, and Igor Liebermann (Stuttgart, Germany) for gene expression analyses.

\section{Authorship Contributions}

Participated in research design: Böhmert, Thomas, Braeuning. Conducted experiments: Tanner, Kubik, Thomas, Hofmann. Performed data analysis: Tanner, Kubik, Thomas, Luckert.

Wrote or contributed to writing of the manuscript: Böhmert, Zanger, Lampen, Braeuning.

\section{References}

Abdel-Razzak Z, Corcos L, Fautrel A, and Guillouzo A (1995) Interleukin-1 beta antagonizes phenobarbital induction of several major cytochromes P450 in adult rat hepatocytes in primary culture. FEBS Lett 366:159-164.

Abdel-Razzak Z, Loyer P, Fautrel A, Gautier JC, Corcos L, Turlin B, Beaune P, and Guillouzo A (1993) Cytokines down-regulate expression of major cytochrome P-450 enzymes in adult human hepatocytes in primary culture. Mol Pharmacol 44:707-715.

Aninat C, Piton A, Glaise D, Le Charpentier T, Langouët S, Morel F, Guguen-Guillouzo C, and Guillouzo A (2006) Expression of cytochromes P450, conjugating enzymes and nuclear receptors in human hepatoma HepaRG cells. Drug Metab Dispos 34:75-83.

Anthérieu S, Rogue A, Fromenty B, Guillouzo A, and Robin MA (2011) Induction of vesicular steatosis by amiodarone and tetracycline is associated with up-regulation of lipogenic genes in HepaRG cells. Hepatology 53:1895-1905.

Araya J, Rodrigo R, Videla LA, Thielemann L, Orellana M, Pettinelli P, and Poniachik J (2004) Increase in long-chain polyunsaturated fatty acid $n-6 / n-3$ ratio in relation to hepatic steatosis in patients with non-alcoholic fatty liver disease. Clin Sci (Lond) 106:635-643.

Braeuning A, Sanna R, Huelsken J, and Schwarz M (2009) Inducibility of drug-metabolizing enzymes by xenobiotics in mice with liver-specific knockout of Ctnnb1. Drug Metab Dispos 37: 1138-1145.

Braeuning A and Schwarz M (2010) beta-Catenin as a multilayer modulator of zonal cytochrome P450 expression in mouse liver. Biol Chem 391:139-148.

Braeuning A, Thomas M, Hofmann U, Vetter S, Zeller E, Petzuch B, Johänning J, Schroth W, Weiss TS, Zanger UM, et al. (2015) Comparative analysis and functional characterization of HC-AFW1 hepatocarcinoma cells: cytochrome P450 expression and induction by nuclear receptor agonists. Drug Metab Dispos 43:1781-1787.

Brown MV, Compton SA, Milburn MV, Lawton KA, and Cheatham B (2013) Metabolomic signatures in lipid-loaded HepaRGs reveal pathways involved in steatotic progression. Obesity (Silver Spring) 21:E561-E570.

Day CP and James OF (1998) Steatohepatitis: a tale of two "hits"? Gastroenterology 114:842-845 Donato MT, Lahoz A, Jiménez N, Pérez G, Serralta A, Mir J, Castell JV, and Gómez-Lechón MJ (2006) Potential impact of steatosis on cytochrome P450 enzymes of human hepatocytes isolated from fatty liver grafts. Drug Metab Dispos 34:1556-1562.

Dubois-Pot-Schneider H, Fekir K, Coulouarn C, Glaise D, Aninat C, Jarnouen K, Le Guével R, Kubo T, Ishida S, Morel F, et al. (2014) Inflammatory cytokines promote the retrodifferentiation of tumor-derived hepatocyte-like cells to progenitor cells. Hepatology 60:2077-2090.

Fierbinteanu-Braticevici C, Baicus C, Tribus L, and Papacocea R (2011) Predictive factors for nonalcoholic steatohepatitis (NASH) in patients with nonalcoholic fatty liver disease (NAFLD). $J$ Gastrointestin Liver Dis 20:153-159.

Finn RD, Henderson CJ, Scott CL, and Wolf CR (2009) Unsaturated fatty acid regulation of cytochrome P450 expression via a CAR-dependent pathway. Biochem J 417:43-54.

Godoy P, Hewitt NJ, Albrecht U, Andersen ME, Ansari N, Bhattacharya S, Bode JG, Bolleyn J, Borner C, Böttger J, et al. (2013) Recent advances in 2D and 3D in vitro systems using primary hepatocytes, alternative hepatocyte sources and non-parenchymal liver cells and their use in investigating mechanisms of hepatotoxicity, cell signaling and ADME. Arch Toxicol 87: 1315-1530.

Gómez-Lechón MJ, Jover R, and Donato MT (2009) Cytochrome p450 and steatosis. Curr Drug Metab 10:692-699.

Hijona E, Hijona L, Arenas JI, and Bujanda L (2010) Inflammatory mediators of hepatic steatosis. Mediators Inflamm 2010:837419.

Honkakoski P and Negishi M (2000) Regulation of cytochrome P450 (CYP) genes by nuclear receptors. Biochem J 347:321-337.

Keller R, Klein M, Thomas M, Dräger A, Metzger U, Templin MF, Joos TO, Thasler WE, Zell A and Zanger UM (2016) Coordinating role of RXR $\alpha$ in downregulating hepatic detoxification during inflammation revealed by fuzzy-logic modeling. PLOS Comput Biol 12:e1004431.

Klein M, Thomas M, Hofmann U, Seehofer D, Damm G, and Zanger UM (2015) A systematic comparison of the impact of inflammatory signaling on absorption, distribution, metabolism, and excretion gene expression and activity in primary human hepatocytes and HepaRG cells. Drug Metab Dispos 43:273-283.

Kugelmas M, Hill DB, Vivian B, Marsano L, and McClain CJ (2003) Cytokines and NASH: a pilot study of the effects of lifestyle modification and vitamin E. Hepatology 38:413-419.

Liu N, Meng Z, Lou G, Zhou W, Wang X, Zhang Y, Zhang L, Liu X, Yen Y, Lai L, et al. (2012) Hepatocarcinogenesis in FXR-/- mice mimics human HCC progression that operates through HNF1 $\alpha$ regulation of FXR expression. Mol Endocrinol 26:775-785.

Luckert C, Hessel S, Lampen A, and Braeuning A (2015) Utility of an appropriate reporter assay: heliotrine interferes with GAL4/upstream activation sequence-driven reporter gene systems. Anal Biochem 487:45-48.

Luckert C, Schulz C, Lehmann N, Thomas M, Hofmann U, Hammad S, Hengstler JG, Braeuning A, Lampen A, and Hessel S (2017) Comparative analysis of 3D culture methods on human HepG2 cells. Arch Toxicol 91:393-406.

McDonald JH (2014) Handbook of Biological Statistics, 3rd ed, Sparky House Publishing, Baltimore, MD

Merrell MD and Cherrington NJ (2011) Drug metabolism alterations in nonalcoholic fatty liver disease. Drug Metab Rev 43:317-334.

Morgan ET, Goralski KB, Piquette-Miller M, Renton KW, Robertson GR, Chaluvadi MR, Charles KA, Clarke SJ, Kacevska M, Liddle C, et al. (2008) Regulation of drug-metabolizing enzymes and transporters in infection, inflammation, and cancer. Drug Metab Dispos 36:205-216.

Naik A, Belič A, Zanger UM, and Rozman D (2013) Molecular interactions between NAFLD and xenobiotic metabolism. Front Genet 4:2.

Pascussi JM, Gerbal-Chaloin S, Pichard-Garcia L, Daujat M, Fabre JM, Maurel P, and Vilarem MJ (2000) Interleukin-6 negatively regulates the expression of pregnane $X$ receptor and 
constitutively activated receptor in primary human hepatocytes. Biochem Biophys Res Commun 274:707-713.

Petzuch B, Groll N, Schwarz M, and Braeuning A (2015) Application of HC-AFW1 hepatocarcinoma cells for mechanistic studies: regulation of cytochrome P450 2B6 expression by dimethyl sulfoxide and early growth response 1. Drug Metab Dispos 43:1727-1733.

Rogue A, Anthérieu S, Vluggens A, Umbdenstock T, Claude N, de la Moureyre-Spire C, Weave RJ, and Guillouzo A (2014) PPAR agonists reduce steatosis in oleic acid-overloaded HepaRG cells. Toxicol Appl Pharmacol 276:73-81.

Rubin K, Janefeldt A, Andersson L, Berke Z, Grime K, and Andersson TB (2015) HepaRG cells as human-relevant in vitro model to study the effects of inflammatory stimuli on cytochrome $\mathrm{P} 450$ isoenzymes. Drug Metab Dispos 43:119-125.

Sanyal AJ, Yoon SK, and Lencioni R (2010) The etiology of hepatocellular carcinoma and consequences for treatment. Oncologist 15 (Suppl 4):14-22.

Schulthess P, Löffler A, Vetter S, Kreft L, Schwarz M, Braeuning A, and Blüthgen N (2015) Signal integration by the CYP1A1 promoter-a quantitative study. Nucleic Acids Res 43:5318-5330.
Toosi AE (2015) Liver fibrosis: causes and methods of assessment, a review. Rom J Intern Med 53 : 304-314.

Weiß J, Rau M, and Geier A (2014) Non-alcoholic fatty liver disease: epidemiology, clinical course, investigation, and treatment. Dtsch Arztebl Int 111:447-452.

Zukunft J, Lang T, Richter T, Hirsch-Ernst KI, Nussler AK, Klein K, Schwab M, Eichelbaum M, and Zanger UM (2005) A natural CYP2B6 TATA box polymorphism (-82T-> C) leading to enhanced transcription and relocation of the transcriptional start site. Mol Pharmacol 67: $1772-1782$.

Address correspondence to: Dr. Albert Braeuning, German Federal Institute for Risk Assessment, Department Food Safety, Max-Dohrn-Str. 8-10, 10589 Berlin, Germany. E-mail: Albert.Braeuning@bfr.bund.de 